\title{
COMPARISON OF DEPRESSION, ANXIETY AND STRESS SCORE AMONG CLINICAL AND NON-CLINICAL HEALTHCARE WORKERS DURING THE COVID-19 PANDEMIC
}

\author{
Mishal Iqbal, Mehak Iqbal*, Nadia Iftikhar**, Syed Hashim Ali Inam***, Hamza Jamil, Shazia Nisar \\ Armed Forces Institute of Cardiology \& National Institute of Heart Diseases/National University of Medical Sciences (NUMS) Rawalpindi Pakistan, *Army \\ Medical College/National University of Medical Sciences (NUMS) Rawalpindi Pakistan, **Pak Emirates Military Hospital/National University of Medical \\ Sciences (NUMS) Rawalpindi Pakistan, ${ }^{* * *}$ Combined Military Hospital Lahore/National University of Medical Sciences (NUMS) Pakistan
}

\section{ABSTRACT}

Objective: To assess the psychological impact of COVID-19 pandemic on healthcare workers.

Study Design: Cross sectional study.

Place and Duration of Study: Armed Forces Institute of Cardiology/National Institute of Health Diseases in June 2020.

Methodology: A total of 317 healthcare professionals, of different designations, affiliated with different public and private sector hospitals in Pakistan participated in this project. They were asked to complete depression, anxiety and stress scales-42 (DASS-42).

Results: Out of 317 healthcare workers, 107 (33.75\%), 111 (35.02\%), 103 (32.49\%) were depressed, anxious and stressed respectively. Female gender was associated with a higher prevalence of depression, anxiety and stress.

Conclusion: More than one third of healthcare workers were depressed, anxious or stressed during the COVID-19 pandemic. Two out of five female healthcare workers were suffering from depression, anxiety or stress.

Keywords: Anxiety, Depression, Healthcare workers, Psychological, Stress.

How to Cite This Article: Iqbal M, Iqbal M, Iftikhar N, Inam SHA, Jamil H, Nisar S. Comparison of Depression, Anxiety and Stress Score Among Clinical and Non-Clinical Healthcare Workers During The COVID-19 Pandemic. Pak Armed Forces Med J 2021; 71(5): 1865-1869. doi: https://doi.org/10.51253/pafmj.v71i5.5372

This is an Open Access article distributed under the terms of the Creative Commons Attribution License (https://creativecommons.org/licenses/by-nc/4.0/), which permits unrestricted use, distribution, and reproduction in any medium, provided the original work is properly cited.

\section{INTRODUCTION}

December 2019, saw the emergence of a deadly and highly infectious acute respiratory syndrome caused by a novel corona virus Severe acute respiratory syndrome coronavirus 2 (SARS-CoV-2) in Wuhan, China. Word Health organization declared this infection to be a pandemic on 11th March 2020. ${ }^{1}$

As of $1^{\text {st }}$ June 2020, over 6.6 million cases have been confirmed worldwide and the death toll has surpassed $375,000 .^{2}$ The frontline workers comprising of medical students, doctors, nurses and medical assistants are at a high risk of contracting the illness. ${ }^{3}$ This pandemic has not only put a strain on the healthcare systems of the world but also on the healthcare workers; physically and psychologically. As with the SARS outbreak in 2003, ${ }^{4}$ the COVID-19 pandemic has also taken its toll on the mental wellbeing of the frontline workers. Mental health is a vital aspect of health. It is more than just the absence of mental illness. ${ }^{5}$ Healthcare professionals are not only exposed to the infection themselves but carry the risk of infecting their loved ones at home, especially those with family members who are elderly, immunocompromised or suffer from chronic medical conditions. A study in China revealed

Correspondence: Dr Mishal Iqbal, Department of Cardiology, Armed Forces Institute of Cardiology, Rawalpindi Pakistan

Received: 20 Nov 2020; revision received: 21 Dec 2020; accepted: 22 Dec 2020 that medical staff were under considerable distress and were provided with mental healthcare during the outbreak of coronavirus. ${ }^{6}$

Increased workload, ${ }^{7}$ physical exhaustion, ${ }^{7}$ mental exhaustion, 7 inadequate personal protective equipment, widespread media coverage, ${ }^{8}$ lack of specific drugs, ${ }^{8}$ the risk of nosocomial transmission, lack of social support, the uncertainty and stigmatization ${ }^{8}$ and risk of infection in relatives all effect the physical and mental wellbeing of healthcare workers. ${ }^{7}$

These mental health problems in healthcare workers in addition to having a lasting impact on the individuals wellbeing, affect decision making capacity, ${ }^{9}$ attention and understanding, ${ }^{9}$ cause increased medical errors and are linked to poor patient care. ${ }^{10}$ Maintaining mental health is essential for better control of infectious diseases 6 in the time of this global pandemic.

A few studies have been carried out regarding the prevalence of depression, anxiety and stress among healthcare professionals during this COVID-19 pandemic all over the world. However, no studies have been done in Pakistan yet to quantify the psychological impact among healthcare workers in this global pandemic. Being diagnosed with depression is considered a social stigma. Our healthcare workers are usually working long hours with poor pay structure and are hesitant in expressing and recognizing their own 
anxiety and depression. There is very little data on the prevalence of depression, stress and anxiety in the time of coronavirus on healthcare workers.

Our primary outcome was to assess the magnitude of the psychological impact in terms of the frequency of depression, anxiety and stress among healthcare workers and our secondary outcome was to compare this between males and females. We also tried to find a relationship between family history of psychiatric disorder and depression, anxiety and stress and to assess the frequency of healthcare workers having suicidal ideation.

\section{METHODOLOGY}

This study was carried out on healthcare professionals of various public and private sector hospitals of Pakistan, in the month of June 2020 the midst of the coronavirus pandemic, to identify healthcare professionals struggling with depression, anxiety and stress.

Inclusion Criteria: Healthcare workers-medical students, house officers, medical officers, postgraduate trainees, consultants, nurses, healthcare administrators, dentists and medical assistants.

Exclusion criteria: Non-consenting individuals were excluded.

Sample size was calculated using WHO sample size calculator taking confidence interval 95\%, margin of error 5\%. A total of 317 healthcare workers participated in this study. Non probability consecutive sampling technique was used. Each of these healthcare workers was categorized into those whose work involved interaction with patients, which included patients of COVID-19 infection and also other inpatients and outpatients and nonclinical-who worked in basic health sciences or medical students who are currently not interacting with patients. The prevalence of depression, anxiety and stress were assigned by distributing by a predesigned, pre validated questionnaire Depression Anxiety Stress Scales 42 (DASS 42), after written informed consent. Depression, anxiety and stress symptoms were assessed on the basis of DASS 42. Personal demographic details, family history of psychiatric disorder and suicidal intent were also recorded. This study was approved by the institutional ethical review board (27/4/R\&D/2020/83).

Data was analyzed using Statistical Pakage for Social Science (SPSS) version-23. Mean and standard deviation were calculated for numerical variables whereas frequency and percentage for categorical variables. The chi square test was used. The $p$-value of $\leq 0.05$ was considered significant.

\section{RESULTS}

A total of 317 healthcare professionals participated in our study, $122(38.5 \%)$ were male and 195 $(61.5 \%)$ were female. The healthcare professionals included $62(19.56 \%)$ consultants, $6(1.89 \%)$ consultants from basic health sciences, $52(16.40 \%)$ post graduate trainees, $28(8.83 \%)$ house officers, $47(14.83 \%)$ medical students, $33(10.41 \%)$ medical officers, $47(14.83 \%)$ nurses, $10(3.15 \%)$ medical assistants, 17 (5.36\%) healthcare administrators and 15 (4.73\%) dentists. The mean age was $33.93 \pm 11.56$ years with the range from 18-80 years.

Of the total participants, $210(66.25 \%)$ din't have depression while $107(33.75 \%)$ were depressed. Out of $25(20.49 \%)$ males were depressed $8(6.55 \%)$ mild, 6 $(4.91 \%)$ moderate, $3(2.45 \%)$ severe and $8(6.55 \%)$ extremely severe] while $97(79.50 \%)$ were not depressed. Among the females, 113 (57.94\%) did not have depression and $82(42.05 \%)$ females were depressed 32 (16.41\%) mild, 18 (9.23\%) moderate, 12 (6.15\%) severe and $20(10.25 \%)$ extremely severe] while $206(64.98 \%)$ participants had no anxiety while 111 (35.02\%) had anxiety. Twenty seven $(22.13 \%)$ males were anxious 7 $(5.73 \%)$ mild, $9(7.37 \%)$ moderate, $2(1.63 \%)$ severe and $9(7.37 \%)$ extremely severe] and $84(43.07 \%)$ females had anxiety $19(9.74 \%)$ mild, 21 (10.76\%) moderate, 18 $(9.23 \%)$ severe and $26(13.33 \%)$ extremely severe] and stress was present in $103(32.49 \%)$ of the healthcare professionals. $24(19.67 \%)$ males 9 (7.37\%) mild, 5 $(4.09 \%)$ moderate, $7(5.73 \%)$ severe and $3(2.45 \%)$ extremely severe and $79(40.51 \%)$ females $22(11.28 \%)$ mild, 20 (10.25\%) moderate, 27 (13.84\%) severe and 10 $(5.12 \%)$ extremely severe] were stressed (Figure-1).

In our healthcare workers, we had 245 (77.3\%) individuals in clinical specialties; involving direct contact with patients and $72(22.7 \%)$ healthcare workers were in nonclinical specialties; not involved in direct patient contact. Our results showed that from the clinical group 72 (29.38\%), 77 (31.42\%) and $73(29.79 \%)$ participants were depressed, anxious and stressed respectively. However, in the nonclinical group 35 (48.61\%), 34 (47.22\%) and 30 (41.66\%) participants were depressed, anxious and stressed respectively. The difference between clinical and nonclinical groups was statistically significant for depression $(p=0.002)$, and anxiety $(p=0.014)$ with the nonclinical group being more effected while the difference between the two groups 
was not statistically significant for stress $(p=0.059)$ shown in Table.

Table: Association of depression, anxiety and stress in clinical and nonclinical healthcare workers.

\begin{tabular}{|c|c|c|c|c|}
\hline \multirow[b]{2}{*}{ Parameters } & & \multicolumn{2}{|c|}{ Health Care Workers } & \multirow[b]{2}{*}{$p$-value } \\
\hline & & Clinical & $\begin{array}{c}\text { Non } \\
\text { Clinical }\end{array}$ & \\
\hline \multirow{2}{*}{ Depression } & Yes & $72(29.38)$ & $35(48.61)$ & \multirow{2}{*}{0.002} \\
\hline & $\mathrm{No}$ & $173(70.62)$ & $37(51.39)$ & \\
\hline \multirow{2}{*}{ Anxiety } & Yes & 77 (31.42) & $34(47.22)$ & \multirow{2}{*}{0.014} \\
\hline & No & $168(68.58)$ & $38(52.78)$ & \\
\hline \multirow{2}{*}{ Stress } & Yes & $73(29.79)$ & $30(41.6)$ & \multirow{2}{*}{0.059} \\
\hline & No & $172(70.21)$ & $42(58.4)$ & \\
\hline
\end{tabular}

Out of 29 (9.1\%) participants had a family history of psychiatric disorders. Out of the 107 depressed participants, $14(13.08 \%)$ participants had a family history of psychiatric disorder while the other 93 (86.92\%) did not have any family history of depression. Of the 111 healthcare workers with anxiety, $15(13.51 \%)$ participants had a family history of psychiatric disorder while $96(86.49 \%)$ did not. Of the 103 participants with stress, $16(15.53 \%)$ had a family history of psychiatric problems while $87(84.47 \%)$ did not. Those with a family history of a psychiatric disorder were more likely to be stressed but not depressed or anxious (Figure-2).

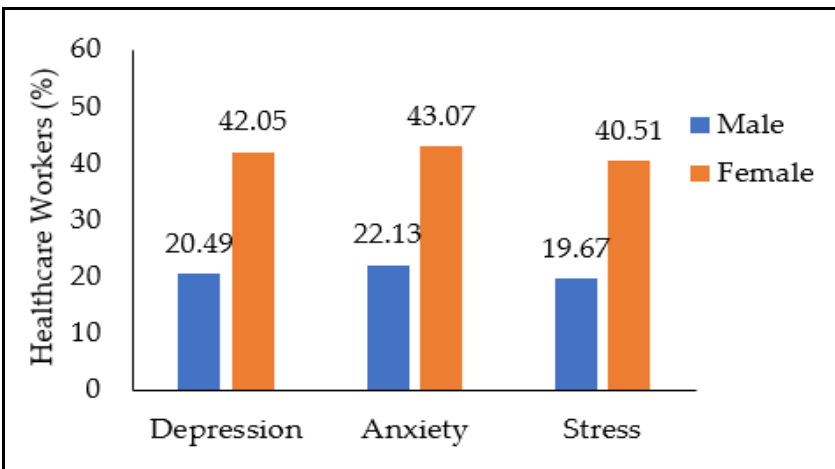

Figure-1:Depression, anxiety and stress in healthcare workers expressed percentages.

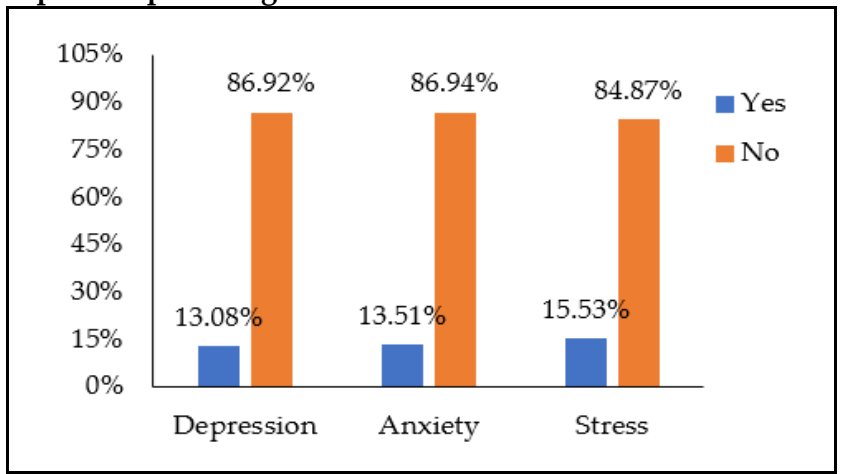

Figure-2: Family history of a psychiatric disorder.
Out of $54(17 \%)$ participants had suicidal ideation whereas $261(82.3 \%)$ did not. 2 of the participants gave no response to this question. None of the participants had attempted suicide.

\section{DISCUSSION}

As of $1^{\text {st }}$ June 2020 , a total of 76,332 confirmed cases of and 1621 deaths from COVID-19, have been reported from Pakistan. ${ }^{11}$ This pandemic, just like any natural disaster may result in anxiety or depression. ${ }^{12}$ Being a healthcare worker during a global pandemic is bound to cause mental health problems. ${ }^{3,7}$ It is thus a priority to monitor the prevalence of depression, anxiety and stress in healthcare workers so as to address these issues and provide support in these difficult times.

Our study revealed that among healthcare workers $33.75 \%$ suffered from depression, $35.02 \%$ from anxiety and $32.49 \%$ from stress. More than one third of all healthcare workers suffered from depression, anxiety and stress. Females at $61.5 \%$ comprised more than half of the healthcare workers. Females were statistically more significantly affected by depression, anxiety and stress, $42.05 \%$ females were depressed, $43.08 \%$ females suffered anxiety while $40.51 \%$ females were stressed.

A study from china ${ }^{8}$ on 1257 healthcare workers found that $60.8 \%$ were nurses, and $39.2 \%$ were physicians; $60.5 \%$ worked in hospitals in Wuhan, and $41.5 \%$ were frontline health care workers. Out of $50.4 \%$ participants reported symptoms of depression, $44.6 \%$ anxiety, $34 \%$ insomnia and $71.5 \%$ distress. Nurses, women, frontline healthcare workers, and those working in Wuhan, China, reported more severe degrees of all measurements of mental health symptoms than other healthcare workers. ${ }^{8}$

A study from Singapore, ${ }^{13}$ on 470 patients found $14.5 \%$ participants screened positive for anxiety, $8.9 \%$ for depression, $6.6 \%$ for stress. This low level could be related to the fact that there were no deaths reported from Singapore at the time this article was written and there was overall mental preparedness and strict infection control measures in place.

A systematic review from Norway, ${ }^{14}$ included 59 studies to examine the psychological effects on clinicians working to manage novel viral outbreaks, reporting on a total of 54,707 healthcare workers. The prevalence of general psychological distress across the studies ranged from 7-97\% (median 37\%), anxiety 9 
90\% (median 24\%), depression 5-51\% (median 21\%), and sleeping problems $34-65 \%$ (median $37 \%$ ). ${ }^{14}$

Another study on anxiety, depression, insomnia and the overall psychological problems in healthcare workers during the COVID-19 pandemic in China showed a prevalence of $46.04 \%, 44.37 \%, 28.75 \%$ and $56.59 \%$, respectively. ${ }^{15}$ The prevalence of the overall psychological problems in physicians, medical residents, nurses, technicians and public health professionals was $60.35 \%, 50.82 \%, 62.02 \%, 57.54 \%$ and $62.40 \%$, respectively. ${ }^{15}$ Frontline healthcare workers had a higher risk of anxiety, insomnia and overall psychological problems as compared to healthcare workers who did not participate in frontline work.

A research on 4679 doctors and nurses from 348 hospitals in 31 provinces of mainland China revealed the prevalence of psychological distress, anxiety symptoms, and depressive symptoms were $15.9 \%, 16 \%$, and $34.6 \% .^{16}$

A systematic review and meta-analysis of data from 10 studies in healthcare workers calculated the pooled prevalence of depression to be $22.8 \%,{ }^{7}$ less than our prevalence. It also found the pooled prevalence of anxiety in 12 studies to be the $23.21 \% .^{7}$ They found the prevalence of depression and anxiety to be higher in females, ${ }^{7}$ as in our study.

Another review on healthcare workers reported extensive strain due to stress as well as depression and anxiety symptoms. ${ }^{17}$ Severe degrees of those symptoms were found in $2.2-14.5 \%$ of participants. ${ }^{17}$

Another study from china on 230 medical frontline workers found the incidence of anxiety was $23.04 \%$ and the incidence in female medical staff was higher than in males $(25.67 \%$ vs $11.63 \%) .{ }^{18}$

An odd observation in our study was that healthcare workers, working directly in contact with patients, were less likely to be depressed, anxious or stressed as compared to those not working in direct contact with patients. It is possible that the extensive media coverage and hype created by social networks contributed to their psychological state.

Clear communication, limitation of shift hours, detailed guidance on the use of personal protective equipment, specialized training on handling of COVID-19 patients can all reduce anxiety caused by the unfamiliarity and uncontrollability of the current situation. It is vital to provide mental health support tailored to individual needs through multidisciplinary teams comprising of mental health professionals.

\section{CONCLUSION}

One out of every three healthcare workers is suffering from depression, anxiety or stress. The prevalence rates for depression, anxiety and stress are significantly higher for female healthcare workers, with $40 \%$ of women in the healthcare team suffering from depression, anxiety or stress.

DASS 42 is in the English language which made it difficult for some groups of healthcare workers to take the survey because of the language barrier.

\section{Conflict of Interest: None.}

\section{Authors' Contribution}

MI: Study design, concept, data analysis, data collection, interpretation drafting, MI: Literature review, questionnaire design, data collection and analysis, drafting, NI: Study concept, literature review, data collection, data analysis, editing, SHI: Questionnaire design, write up, data collection and analysis, HJ: Study concept, literature review, data interpretation and editing, SN: Data collection interpretation analysis and drafting.

\section{REFERENCES}

1. Organization WH. WHO Director-General's opening remarks at the media briefing on COVID-19-11 March 2020 [Internet] Available from: https://www.who.int/dg/speeches/detail/ who-director-general-s-opening-remarks-at-the-media-briefingon-COVID-19---11-march-2020 [Accessed on June 11, 2020].

2. The Space Report; CNN. June 1 coronavirus news 2020. [Internet] Available from: https://edition.cnn.com/world/live-news/ coronavirus-pandemic-06-01-20-intl/index.html [Accessed on June 11, 2020].

3. Sim MR. The COVID-19 pandemic: major risks to healthcare and other workers on the front line. Occup Environ Med 2020; 77(5): 281-282.

4. Wu P, Fang Y, Guan Z, Fan B, Kong J, Yao Z, et al. The psychological impact of the SARS epidemic on hospital employees in China: exposure, risk perception, and altruistic acceptance of risk. Can J Psych 2009; 54(5): 302-311.

5. WHO. Mental health: strengthening our response. [Internet] Available from: https://www.who.int/news-room/fact-sheets/ detail/mental-health-strengthening-our-response [Accessed on June 11, 2020].

6. Chen Q, Liang M, Li Y, Guo J, Fei D, Wang L, et al. Mental health care for medical staff in China during the COVID-19 outbreak. Lancet Psych 2020; 7(4): e15-e16.

7. Pappa S, Ntella V, Giannakas T, Giannakoulis VG, Papoutsi E, Katsaounou P. Prevalence of depression, anxiety, and insomnia among healthcare workers during the COVID-19 pandemic: A systematic review and meta-analysis. Brain Behav Immun 2020; 88(2): 901-907.

8. Lai J, Ma S, Wang Y, Cai Z, Hu J, Wei N, et al. Factors associated with mental health outcomes among health care workers exposed to coronavirus disease 2019. JAMA Netw Open 2020; 3(3): e203976.

9. Kang L, Li Y, Hu S, Chen M, Yang C, Yang BX, et al. The mental health of medical workers in Wuhan, China dealing with the 2019 novel coronavirus. Lancet Psych 2020; 7(3): e14-e18.

10. Coentre R, Faravelli C, Figueira ML. Assessment of depression and suicidal behaviour among medical students in Portugal. Int J Med Educ 2016; 7(1): 354-363. 


\section{Psychological Impact of COVID-19 Pandemic}

11. Pakistan Go. Pakistan cases details 2020, [Internet] Available from: http://COVID.gov.pk/stats/pakistan. [Accessed in June 20, 2020]

12. Pfefferbaum B, North CS. Mental health and the COVID-19 pandemic. N Engl J Med 2020; 383(6): 510-512.

13. Tan BY, Chew NW, Lee GK, Jing M, Goh Y, Yeo LL, et al. Psychological impact of the COVID-19 pandemic on health care workers in Singapore. Ann Intern Med 2020; 1(1): M201083.

14. Muller AE, Hafstad EV, Himmels JPW, Smedslund G, Flottorp S, Stensland S, et al. The mental health impact of the COVID-19 pandemic on healthcare workers, and interventions to help them: a rapid systematic review. medRxiv. Available from: https:// www.sciencedirect.com/science/article/pii/S0165178120323271 [Accessed in June 22, 2020]
15. Ng QX, De Deyn MLZQ, Lim DY, Chan HW, Yeo WS. The wounded healer: A narrative review of the mental health effects of the COVID-19 pandemic on healthcare workers. Asian J Psychiatr 2020; 54(2): 102258.

16. Liu Z, Han B, Jiang R, Huang Y, Ma C, Wen J, et al. Mental health status of doctors and nurses during COVID-19 epidemic in China. [Internet] Available from: https://papers.ssrn.com/sol3/ papers.cfm?abstract_id=3551329. [Accessed in June 24, 2020]

17. Bohlken J, Schömig F, Lemke MR, Pumberger M, Riedel-Heller SG. COVID-19 pandemic: stress experience of healthcare workers-a short current review. Psychiatr Prax 2020; 47(4): 190-197.

18. Huang J, Han M, Luo T, Ren A, Zhou X. Mental health survey of 230 medical staff in a tertiary infectious disease hospital for COVID-19. Zhonghua Lao Dong Wei Sheng Zhi Ye Bing Za Zhi 2020; 38(3): 192-195. 